\title{
MicroRNAs and DNA methylation as epigenetic regulators of mitosis, meiosis and spermiogenesis
}

\author{
Chencheng Yao ${ }^{1, *}$, Yun Liu ${ }^{1, *}$, Min Sun ${ }^{1}$, Minghui Niu ${ }^{1}$, Qingqing Yuan ${ }^{1}$, Yanan Hai ${ }^{1}$, Ying Guo $^{1}$, \\ Zheng Chen ${ }^{1}$, Jingmei Hou ${ }^{1}$, Yang Liu ${ }^{1}$ and Zuping $\mathrm{He}^{1,2,3,4}$ \\ ${ }^{1}$ State Key Laboratory of Oncogenes and Related Genes, School of Medicine, Renji-Med X Clinical Stem Cell Research \\ Center, Ren Ji Hospital, Shanghai Jiao Tong University, 160 Pujiang Road, Shanghai 200127, China, ${ }^{2}$ Department of \\ Urology, School of Medicine, Shanghai Institute of Andrology, Ren Ji Hospital, Shanghai Jiao Tong University, \\ Shanghai Human Sperm Bank, 145 Shangdong Road, Shanghai 200001, China, ${ }^{3}$ Shanghai Key Laboratory of Assisted \\ Reproduction and Reproductive Genetics, Shanghai 200127, China and ${ }^{4}$ Shanghai Key Laboratory of Reproductive \\ Medicine, Shanghai 200025, China
}

Correspondence should be addressed to Z He; Email: zupinghe@sjtu.edu.cn

*(C Yao and Y Liu contributed equally to this work)

\begin{abstract}
Spermatogenesis is composed of three distinctive phases, which include self-renewal of spermatogonia via mitosis, spermatocytes undergoing meiosis I/II and post-meiotic development of haploid spermatids via spermiogenesis. Spermatogenesis also involves condensation of chromatin in the spermatid head before transformation of spermatids to spermatozoa. Epigenetic regulation refers to changes of heritably cellular and physiological traits not caused by modifications in the DNA sequences of the chromatin such as mutations. Major advances have been made in the epigenetic regulation of spermatogenesis. In this review, we address the roles and mechanisms of epigenetic regulators, with a focus on the role of microRNAs and DNA methylation during mitosis, meiosis and spermiogenesis. We also highlight issues that deserve attention for further investigation on the epigenetic regulation of spermatogenesis. More importantly, a thorough understanding of the epigenetic regulation in spermatogenesis will provide insightful information into the etiology of some unexplained infertility, offering new approaches for the treatment of male infertility.

Reproduction (2015) 150 R25-R34
\end{abstract}

\section{Introduction}

Epigenetic regulation refers to the inheritable changes of phenotype or gene expression caused by mechanisms other than modifications in the underlying DNA sequences (Egger et al. 2004). Epigenetic regulators can control the expression of genes at the transcriptional and/or post-transcriptional levels, and epigenetic disorders may lead to human diseases, including mental retardation and cancer. Formation of functional spermatozoa is a complex process which involves self-renewal of spermatogonia by mitosis, meiosis of spermatocytes to form haploid spermatids, and transformation of spermatids to spermatozoa via spermiogenesis. These orchestrated processes involve unique transcriptional regulation and comprehensive chromosome remodeling. To keep the totipotence of zygote, epigenetic markers of male germ cells must be reset. Consequently, male germ cells are particularly vulnerable to epigenetic defects. Thus, epigenetic abnormality could be a potential cause of male infertility. The epigenetic reprogramming includes primordial germ cell (PGC) specification, spermatogonial stem cell (SSC) renewal, chromosome remodeling in the meiosis and histone transition in sperm maturation.

There are several major epigenetic regulators, including small non-coding RNAs and DNA methylation. Notably, Human Genome Program indicates that genes encoding proteins comprise only about $2 \%$ of human genomics, whereas the remainder consists of non-coding nucleic acid. MicroRNA (miRNA) is a novel class of endogenous small RNA molecules ( $\sim 18-22$ nucleotides in length) that can negatively control their targeting genes. MiRNAs are initially transcribed by RNA endogenous polymerase as primary miRNAs and eventually incorporated into the miRNA-induced silencing complex (miRISC) under a series of processing steps (Bartel 2004). It has been demonstrated that miRNAs are involved in the mitotic, meiotic and postmeiotic phases of spermatogenesis by inhibiting the expression of target genes (Tang et al. 2007, Hayashi et al. 2008). DNA methylation refers to the process in 
which a methyl group is added to the cytosine nucleotide with the typical location in the symmetric CG contexts (Chen \& Li 2004), and it is related to genomic imprinting, transposon repression and $\mathrm{X}$-chromosome inactivation in normal development. DNA methylation defects are associated with infertility in humans. With the application of 5-aza-2'-deoxycytidine, a demethylation agent, aberrant male germ cell development has been detected in males with reduced fertility (Kelly et al. 2003). Therefore, a thorough understanding of epigenetic regulation in spermatogenesis is of great significance for the therapy of male infertility and for developing new approaches for male contraception. Based on recent advances in epigenetic regulation, we discuss herein the roles of miRNAs and DNA methylation in regulating mitosis, meiosis and spermiogenesis.

\section{MicroRNA and spermatogenesis}

MiRNAs are small non-coding regulatory RNA molecules that regulate gene expression either by targeting mRNA for degradation or by translation inhibition, as we illustrated in Fig. 1. In general, mature miRNA is processed through at least three sequential steps: i) the pre-miRNA ( $\sim 70$ nucleotides) is generated by the primary transcripts (pri-miRNA) through RNase III member Drosha and its cofactors Pasha/DGCR8 (Lee et al. 2003, Han et al. 2004); ii) the pre-miRNA is transported from the nucleus to the cytoplasm by

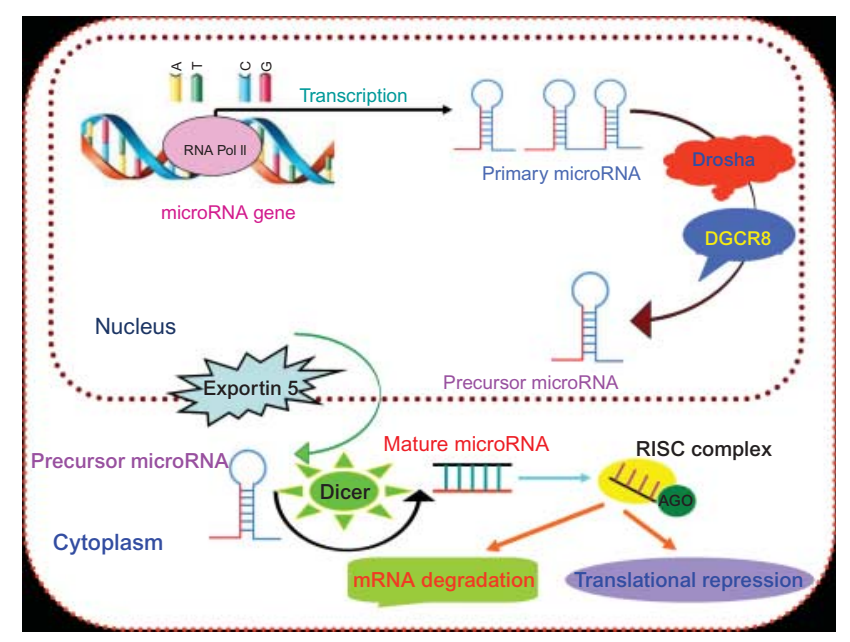

Figure 1 Schematic diagram shows miRNA biogenesis. MiRNA genes are transcribed as the primary capped and polyadenylated precursors of miRNA (pri-miRNAs) by RNA polymerase II in the nucleus, and the pri-miRNAs are further cleaved by Drosha and DGCR8. The processing of the pri-miRNAs by RNase III enzyme Drosha along with cofactor DGCR8 gives rise to the stem-loop pre-miRNA that is exported by Exportin 5 from the nucleus to the cytoplasm. In the cytoplasm, the RNase III enzyme Dicer catalyzes the pre-miRNA to form mature miRNAs. The mature miRNAs are incorporated into the RNA-induced silencing complex (RISC) that guides the $3^{\prime}$-UTR of the target gene. The association of the miRNA-RISC results in the silencing of the target gene by mRNA degradation or translational repression.
Exportin 5 (Lund et al. 2004); and iii) the mature miRNAs are generated by Dicer cleavage (Hutvagner et al. 2001).

MiRNA interacts with the targeting genes through specific base-pairing with the key domain of the mature miRNA, particularly at bases $2-8$ of the $5^{\prime}$-end known as the 'seed' region. The mRNA is cleaved under the condition of perfect base-pairing between miRNAs and miRNA regulatory elements (MRE) in the $3^{\prime} U T R$ of targeting mRNAs, whereas imperfect interaction between the miRNA and MRE leads to translational repression. The first discovered miRNA, namely, lin-4, acts as a posttranscriptional regulator (Lee et al. 1993, Wightman et al. 1993). Since then, a number of studies have shown that miRNAs are involved in various kinds of biological processes, including development, virus defense, hematopoiesis, organ formation, cell proliferation and apoptosis. Growing evidence has indicated that miRNAs play critical roles in regulating male germ cell development and are essential for epigenetic regulation of the mitosis, meiosis and spermiogenesis (Hayashi et al. 2008, Maatouk et al. 2008, Huszar \& Payne 2013).

\section{The roles of miRNAs in SSC self-renewal and differentiation}

SSCs are located in the basal compartment of the seminiferous epithelium, and they play important roles in maintaining normal spermatogenesis and the transmission of genetic information to next generations. To date, studies on SSCs have been reported in other aspects, such as SSC transplantation, long-term culture of SSCs and transgenic animals (Brinster \& Avarbock 1994, Khaira et al. 2005, Sadri-Ardekani et al. 2009, Kanatsu-Shinohara \& Shinohara 2013). Significant advances have been made in the field of SSC biology, including gene regulation, signaling pathways and epigenetic regulators. Interestingly, a series of studies have demonstrated that miRNAs play essential roles in conferring mouse SSC fate determinations.

In the past several years, a number of miRNA expression profiles have been identified in the murine testes by miRNA microarrays, RT-PCR or small RNA sequencing technology (Hayashi et al. 2008, Buchold et al. 2010, Jung et al. 2010). The expression profiling of interesting miRNAs in the testis has been outlined in Table 1, and their potential roles in regulating SSC selfrenewal and differentiation are illustrated in Fig. 2. In total, 141 miRNAs have been identified in mouse testis, of which 29 are novel by small RNA sequencing method (Ro et al. 2007). These miRNAs may be involved in mouse spermatogenesis. It has been reported that miR21 is functionally important in regulating SSC (Thy $1^{+}$) population by working with ETV5, a critical transcription factor for maintaining the self-renewal of SSCs (Niu et al. 2011). Meanwhile, miR-34c, miR-182, miR-183 and miR-146a have been shown to be preferentially expressed in the SSC (Thy $1^{+}$)-enriched population 
Table 1 Expression and roles of miRNAs in mammalian testis.

\begin{tabular}{|c|c|c|}
\hline microRNA & Expression and roles & References \\
\hline miR-17-92 and miR-290-295 clusters & miR-17-92 and miR-290-295 clusters are highly expression in spermatogonia & Hayashi et al. (2008) \\
\hline miR-141, -200a, -200c and -323 & $\begin{array}{l}\text { Expression of miR-141, }-200 a,-200 c \text { and }-323 \text { is reduced during PGC } \\
\text { development }\end{array}$ & Hayashi et al. (2008) \\
\hline let- $7 \mathrm{a}, \mathrm{d}, \mathrm{e}, \mathrm{f}$ and $\mathrm{g}$ & Expression of let-7a, $d, e, f$ and $g$ is increased during PGC development & Hayashi et al. (2008) \\
\hline miR-34c, $-182,-183$ and $-146 a$ & $\begin{array}{l}\text { miR-34c, }-182,-183, \text { and }-146 \text { a are preferentially expressed in } \\
\text { Thy } 1^{+} \text {-enriched population }\end{array}$ & Niu et al. (2011) \\
\hline miR-21 & miR-21 is important in maintaining mouse Thy $1^{+}$population & Niu et al. (2011) \\
\hline miR-20 and miR-106a & $\begin{array}{l}\text { miR-20 and miR-106a regulate mouse SSC renewal by targeting STAT3 and } \\
\text { Ccnd1 }\end{array}$ & He et al. (2013) \\
\hline miR-135a & miR-135a contributes to the maintenance of mouse SSC by regulating FoxO1 & Moritoki et al. (2014) \\
\hline miR-221 and 222 & $\begin{array}{l}\text { miR-221 and } 222 \text { play an important role in the maintenance of the } \\
\text { undifferentiated state of mouse spermatogonia }\end{array}$ & Yang et al. (2013) \\
\hline miR-17-92 cluster and miR-106b-25 & $\begin{array}{l}\text { miR-17-92 cluster and miR-106b-25 are involved in the regulation of mouse } \\
\text { spermatogonial differentiation }\end{array}$ & Tong et al. (2012) \\
\hline Mirlet7 family & $\begin{array}{l}\text { let-7 family miRNAs play a role in retinoic acid-induced spermatogonial } \\
\text { differentiation }\end{array}$ & Tong et al. (2011) \\
\hline miR-146 & miR-146 regulates spermatogonial differentiation by retinoic acid & Huszar \& Payne (2013) \\
\hline miR-449/, miR-34b/c & miR-449 and miR-34b/c are located in mouse spermatocytes and spermatids & $\begin{array}{l}\text { Bouhallier et al. (2010), } \\
\text { Bao et al. (2012) and } \\
\text { Wu et al. (2014) }\end{array}$ \\
\hline miR-34a, $-34 b$ and $-34 c$ & miR-34a, $-34 b$ and $-34 c$ are expressed in bovine sperm & Tscherner et al. (2014) \\
\hline miR-34c & $\begin{array}{l}\text { miR-34c is highly expressed in mouse pachytene spermatocytes and } \\
\text { round spermatids }\end{array}$ & Liang et al. (2012) \\
\hline miR-469 & miR-469 expression is associated with sperm development & Dai et al. (2011) \\
\hline miR-122 & miR-122 expression is involved in sperm development & Yu et al. (2005) \\
\hline
\end{tabular}

(Niu et al. 2011), reflecting potential roles of these miRNAs in mediating self-renewal and maintenance of mouse SSCs. On the other hand, miR-34c has been demonstrated to be involved in the differentiation of mouse SSCs via targeting Nanos2 (Yu et al. 2014). Taken together, miR-34 seems to be associated with both division and differentiation of SSCs through targeting different genes. Moreover, miR-135a has been shown to retain the undifferentiated state of SSCs via FoxO1 (Moritoki et al. 2014). Impairing the function of $X$ chromosome-clustered miR-221/222 in mouse undifferentiated spermatogonia causes a transition from $\mathrm{KIT}^{-}$to $\mathrm{KIT}^{+}$state and the loss of stem cell ability to repopulate spermatogonia (Yang et al. 2013). This study suggests that miR-221/222 might play an important role in the maintenance of the undifferentiated state of mammalian spermatogonia. In addition, the expression of miR-17-92 (Mirc1) and miR-106b-25 (Mirc3) clusters has been shown to be significantly down-regulated by retinoic acid induction (Tong et al. 2012). During the development of male germ cells, miR-17-92 and miR-290-295 cluster are highly expressed in mouse spermatogonia (Hayashi et al. 2008). Collectively, these findings suggest that miR-17-92 clusters play potential roles in maintaining the stemness of SSCs. Consistent with these observations, we have recently demonstrated that miR20 and miR-106a are required for the proliferation and survival of mouse SSCs through targeting Stat3 and Ccnd1 (He et al. 2013).

SSC differentiation is an essential step in spermatogenesis, which has been found to be modulated by miRNAs. The expression of miR-141, miR-200a, miR-200c and miR-323 are down-regulated during male germ cell development, whereas the levels of let-7 family (let-7a, $\mathrm{d}, \mathrm{e}, \mathrm{f}$ and $\mathrm{g}$ ) are up-regulated (Hayashi et al. 2008), indicating distinct roles of these miRNA in male germ cell differentiation. This speculation has been verified by the findings that the members of miRNA let-7 family (e.g., let-7a/b/c/d/e) regulate mouse SSC differentiation (Tong et al. 2011, 2012). MiR-146 is involved in mouse

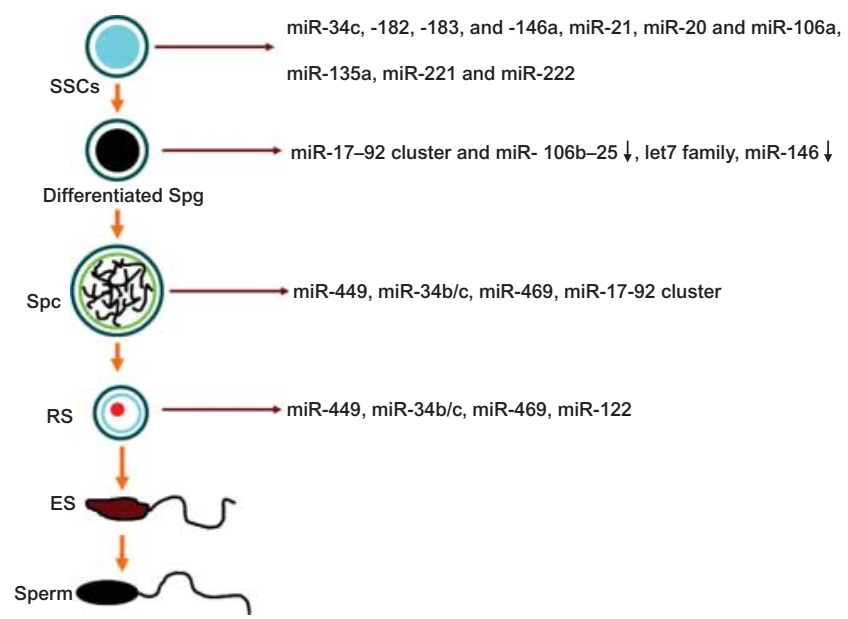

Figure 2 The expression patterns of miRNAs in various types of cells in mammalian testis. MiR-17-92 and miR-290-295 cluster are highly expressed in mouse spermatogonia, and numerous miRNAs (e.g. miR-34c, -182, -183 and -146a, miR-21, miR-20, miR-106a, miR-135a, miR-221, and miR-222) have been shown to regulate SSC self-renewal. In contrast, miR-17-92 cluster, miR-106b-25, let-7 family and miR-146 are involved in the regulation of mouse spermatogonial differentiation. MiR-449, miR-34b/c and miR-469 are located in mouse spermatocytes and spermatids, while miR-34a, $-34 b,-34 c$ and miR-122 are associated with sperm development. SSCs, spermatogonial stem cells; Spc, spermatocytes; RS, round spermatids; ES, elongating spermatids. 
SSC differentiation induced by retinoic acid signaling, because its expression is diminished by $\sim 180$-fold in differentiating spermatogonia vs undifferentiated spermatogonia by targeting mediator complex subunit 1 , a coregulator of retinoid receptors (RARs and RXRs) (Huszar \& Payne 2013). In addition to regulating the differentiation, Mir-17-92 (Mirc1) cluster also protects meiotic cells from apoptosis by down-regulating E2F1 translation (Novotny et al. 2007). Although numerous miRNAs have been found in modulating the self-renewal and differentiation of SSCs in rodents, there are no reports in the literature supporting miRNA function in human SSCs due to difficulties in obtaining human testis tissues in particular human SSCs. We have recently compared the global miRNA profiles among human spermatogonia, pachytene spermatocytes and round spermatids, illustrating that 110 miRNAs are differentially expressed between human spermatogonia and pachytene spermatocytes (Liu et al. 2015), suggesting that these miRNAs are involved in mitosis and meiosis.

\section{Functions of miRNAs in meiosis and spermiogenesis}

Meiosis of spermatocytes and spermiogenesis of spermatids are two indispensable phases of spermatogenesis through which functional male gametes are generated. These intricate processes must be tightly regulated, and any mistake in these processes leads to the abnormality of spermatogenesis. Some unexplained male infertility may be the result of meiotic arrest in spermatocytes and/or defects of spermiogenesis in post-meiotic spermatids. Notably, miRNAs likely play a role in regulating gene expression throughout these developmental stages. Drosha is required for the biogenesis of miRNAs in spermatogenesis, and conditional knockout of Drosha in male germ cells leads to a significant decrease of miRNAs, reduces testis weight and a severe disruption in both meiotic and haploid phases of spermatogenesis (Wu et al. 2012). Meanwhile, Dicer is essential for the biogenesis of both miRNAs and siRNAs in spermatogenesis. After conditional knockout of miRNA maturationrelated key enzyme Dicer in testes, the number of mouse SSCs is decreased and their differentiation is also blocked. In addition, loss of Dicer1 results in male infertility in mice (Maatouk et al. 2008), illustrating an essential role of miRNAs in spermatogenesis. Sertoli cellspecific deletion of Dicer, a central component of the RNAi machinery, severely impairs Sertoli cell competence, leading to male infertility due to the absence of mature spermatozoa and testicular degeneration (Papaioannou et al. 2009), reflecting an important role of the Dicer for male germ cell development. Germ cellspecific deletion of Dicer1 leads to overexpression of genes for meiotic sex chromosome inactivation (MSCl), an increase in spermatocyte apoptosis and defects in chromatin organization and nuclear shaping of elongating spermatids (Korhonen et al. 2011, Romero et al.
2011, Greenlee et al. 2012, Zimmermann et al. 2014), suggesting that Dicer1 is required for the meiotic and haploid phases of spermatogenesis. In addition to Dicer, DGCR8 has been shown to be indispensable for the biogenesis of miRNAs but not endo-siRNAs, and similar symptom occurs in the conditional DGCR8-knockout mice, although the phenotype is less severe compared to the Dicer1-knockout mice (Zimmermann et al. 2014). Moreover, the knockout study of canonical enzymes in the miRNA biogenesis process reveals that miRNAs are of great importance in meiosis and spermiogenesis phases of spermatogenesis since spermatogonial differentiation appears to be unaffected by canonical enzyme deficiency. These findings suggest that miRNAs necessary to confer spermatogonial differentiation may be synthesized by non-canonical enzymes, and the underlying mechanism remains to be elucidated.

It has recently been found that miR-449 is predominantly expressed in mouse testes and it is mainly located in spermatocytes and spermatids (Bao et al. 2012, Wu et al. 2014). Interestingly, miR-34b and miR-34c have been found to resemble the 'seed' sequence of miR-449. Coincidentally, miR-34b and miR-34c exhibit a similar effect to that of miR-449 during the development of male germ cells and spermatogenesis (Bouhallier et al. 2010, Bao et al. 2012, Wu et al. 2014). Individual deficiency in $\mathrm{miR}-34 \mathrm{~b} / \mathrm{c}$ or miR-449 appears to have no obvious effect; however, simultaneous inactivation of miR-34b/34c and miR-449 leads to mouse oligoasthenoteratozoospermia (Wu et al. 2014), implicating that double or triple knockout approach of miRNAs is needed to obtain the phenotype for certain miRNAs. It has been demonstrated that all the members of miR-34 family (i.e. miR-34a, $-34 b$ and $-34 c$ ) are found in bovine sperm (Tscherner et al. 2014). Additionally, miR-34c seems to be important for the later steps of spermatogenesis because it is highly expressed in mouse pachytene spermatocytes and round spermatids in murine testis by targeting the activating transcription factor 1 (ATF1; Liang et al. 2012). Moreover, the induction of ATF1 in spermatocytes of the pachytene stage is essential for maintaining cell viability and mouse early development (Bleckmann et al. 2002, Persengiev \& Green 2003). These findings illustrate that the members of miR-34 family are vital for spermatogenesis. The expression of transition protein (TP) and protamine (Prm) proteins are required for spermatid elongation and spermiogenesis. MiR-469 has been found to be essential for the timely translation at later stages of spermiogenesis by inhibiting of TP2 and Prm2 transcripts (Dai et al. 2011). Another miRNA, namely miR-122, has been shown to reduce the expression of TP2 at transcription level by mRNA cleavage (Yu et al. 2005). These studies illustrate that miR-469 and miR-122 are associated with spermiogenesis. MiR-18 is abundantly expressed in the testis and it displays cell type-specific expression, with the highest intensity in spermatocytes. Meanwhile, miR-18 directly targets heat 
shock factor 2, which is a key transcription factor essential for influencing embryogenesis and gametogenesis (Bjork et al. 2010).

Collectively, miRNAs play essential roles in regulating each step of male germ cell development, including mitosis, meiosis and spermiogenesis in rodents. Nevertheless, it remains to be defined whether miRNAs are required for spermatogenesis in humans. Recently, we have identified 559 miRNAs distinctively expressed by human spermatogonia, pachytene spermatocytes and round spermatids (Liu et al. 2015). Using miRNA microarray, we have identified 144 miRNAs that are significantly up-regulated while 29 miRNAs are downregulated in pachytene spermatocytes vs round spermatids (Liu et al. 2015), reflecting essential roles of these miRNAs in mediating spermiogenesis. A number of novel binding targets of the differentially expressed miRNAs have been identified using various bioinformatics software and verified by real time PCR (Liu et al. 2015). Significantly, our ability to unveil the global distinct miRNA signatures and binding targets of human spermatogonia, pachytene spermatocytes and round spermatids could provide novel small RNA regulatory mechanisms mediating three stages of human spermatogenesis and offers new targets for treatment of male infertility.

\section{DNA methylation and spermatogenesis}

DNA methylation, by definition, is an epigenetic modification that DNA methyltransferase transfers a methyl unit from the $S$-adenosyl-L-methionine to the 5th position of cytosine residues in nucleotides (Chen \& Li 2004, Portela \& Esteller 2010). DNA methylation occurs in the cytosine-phosphate-guanine dinucleotides (CpGs) context, although it is also located in few nonCpG sites; however, the function of DNA methylation situated in non-CpG sites remains unclear (Jones 2012). Dinucleotides with high frequency of CpG palindromes are called $\mathrm{CpG}$ islands (CGI). The $\mathrm{CpG}$ island is defined as the DNA region whose $\mathrm{G} \& \mathrm{C}$ content should be equal to or $>55 \%$ of the base pairs, and the ratio of observedto-expected CpGs is over 0.65 (Takai \& Jones 2002). Normally, CpG islands located in transcription start sites (TSS) are unmethylated in developmental and housekeeping genes, and the active TSS is marked by the trimethylation of histone $\mathrm{H} 3$ lysine 4 (H3K4me3) (Thomson et al. 2010, Messerschmidt et al. 2014). The CGI promoter can be repressed by various molecular strategies. On one hand, CGI promoter undergoes de novo methylation and it can be kept in methylated state by maintaining methyltranferase activity. This mechanism is stable and lasts for a long period, and it is responsible for $\mathrm{X}$ chromosome inactivation and genomic imprinting (Jones \& Liang 2009). On the other hand, CGI promoter can be repressed by a large protein complex through binding to the target genes so that the chromatin is enclosed (Boyer et al. 2006). Its target gene is marked with repressive and active $\mathrm{H} 3 \mathrm{~K} 4 \mathrm{me} 3$, which is called the bivalent modification pattern (Bernstein et al. 2006, Pan et al. 2007). This strategy is unstable and the silenced genes can be reactivated under certain circumstances (Cedar \& Bergman 2009). The CpG islands located in gene bodies are marked by H3K9me3 and they bind to MeCP2 which is associated with histone deacetylation and chromatin condensation to repress gene expression (Nguyen et al. 2001). In contrast to CpG islands located in the TSS, methylation of $\mathrm{CpG}$ islands located in gene bodies is able to activate genes (Jones 1999).

There are two patterns of DNA methylation, namely, the maintenance methylation and the de novo methylation (Holliday \& Pugh 1975). A number of DNA methyltransferases, including DNMT1, DNMT1o, DNMT3a, DNMT3b and DNMT3L, are involved in these two mechanisms. DNMT1 is responsible for maintenance methylation activity and it has a high affinity for hemi-methylated DNA (Pradhan et al. 1999). It is composed of C-terminal methyltransferase domain and N-terminal regulation domain (Song et al. 2012). $\mathrm{N}$-terminal domain interacts with proliferating cell nuclear antigen (PCNA; Chuang et al. 1997) and UHRF1, which facilitates DNMT1 binding to the replication fork (Bostick et al. 2007, Hashimoto et al. 2008). DNMT1 recognizes the hemi-methylated DNA and methylates the newly generated strand so that the DNA methylation patterns can be maintained (Cedar \& Bergman 2012). DNMT3a and DNMT3b are responsible for the de novo methylation (Okano et al. 1998). After the wave of demethylation in PGCs, the imprinted genes and transposon methylation patterns can be reestablished through the de novo methylation (Law \& Jacobsen 2010). The de novo methylation mechanism is interpreted by both histone modification and piRNA pathways (Ooi et al. 2007). As an example, H3K4 methylation may be involved in de novo methylation. DNMT3L lacking the methyltransferase activity (Chedin et al. 2002) can recruit DNMT3a2 to the unmethylated H3K4 tail site (Jia et al. 2007), whereas this activity is inhibited by the methylation of the H3K4 tails (Ooi et al. 2007). Consequently, only the unmethylated $\mathrm{H} 3 \mathrm{~K} 4$ leads to the de novo methylation. Additionally, piRNA pathways may also explicate de novo methylation, since piRNA is related to the transposon silencing and the silenced transposons are methylated through de novo methylation (Aravin et al. 2007). PiRNA can interact with PIWI proteins, including MILI and MIWI. Notably, male germ cells with MILI and MIWI2 deficiency assume DNA methylation defect in retrotransposons regulation regions in the phase of de novo methylation (Kuramochi-Miyagawa et al. 2008). Meanwhile, piRNA-PIWI complex could indirectly recruit the de novo methyltransferase through interacting with chromatin modifiers (Aravin \& Bourc'his 2008). Together, these findings indicate that the 
de novo methylation regulates the development of male germ cells.

There are also two different modes for demethylation, namely the passive and active demethylation. Aberrant maintenance of methylation pathways in the replication may decipher the passive demethylation which is replication-dependent (Mertineit et al. 1998, Kagiwada et al. 2013). Meanwhile, the active demethylation is likely an enzymatic process. First of all, $5 \mathrm{mC}$ is hydroxylated by ten-eleven translocation enzyme to become $5 \mathrm{hmC}$ and further to 5 -formylcytosine $(5 \mathrm{fC})$ or 5-carboxycytosine (5caC) (He et al. 2011, Ito et al. 2011). Secondly, the modified $5 \mathrm{mC}$ can be recognized by the activation-induced deaminase (Aid) or apolipoprotein B mRNA-editing enzyme catalytic polypeptide 1 (Apobec1) and converted to thymidine, which results in $\mathrm{T}: \mathrm{G}$ mismatch. After the removal of the T:G mismatch by the glycosylase thymine-DNA glycosylase and methylCpG binding domain protein 4 , the site can be repaired by a base pair excision repair mechanism to regenerate the unmethylated cytosine (Rai et al. 2008, Cortellino et al. 2011).

\section{Bimodal pattern of DNA methylation in male germ cells}

As illustrated in Fig. 3, DNA methylation in male germ cell specification and maturation displays a bimodal pattern. PGC, the origin of male germ cells, derives from epiblast at E6.5-E7.5. Once stimulated by BMP4, PGCs migrate from the epiblast to hindgut at E7.5-E9 and the genital ridge at E9.5-E11.5 (Saitou \& Yamaji 2012). In the E6.5 mouse embryo, the DNA patterns in PGCs are similar to those in somatic cells in term of their fates. For instance, the pluripotent markers are repressed by DNA hypermethylation (Seisenberger et al. 2012). During the migration of PGCs, the epigenetic marks are widely erased (Popp et al. 2010). In particular, the paternal imprinted genes and transposons of PGCs are also demethylated. The re-establishment of DNA methylation patterns in male germ cells initiates from the prospermatogonia or gonocytes. Although a great proportion of DNA methylation is acquired during the prenatal mitotic arrest of the gonocytes, the de novo methylation and maintenance methylation occur in the mitosis of spermatogonia and meiotic prophase I, while the maintenance methylation appears only in mitosis (Santos et al. 2005, Oakes et al. 2007).

The global erasure of the DNA methylation also occurs during early embryonic development (Mayer et al. 2000, Oswald et al. 2000). The outcomes and gene expression patterns of demethylation may differ between early embryogenesis and PGCs. First, the demethylation during the early embryogenesis results in totipotency, whereas the demethylation of the PGCs derived from the epiblast leads to pluripotency. Secondly, the demethylation manner during the early embryogenesis is similar to the process of demethylation in PGCs with
A

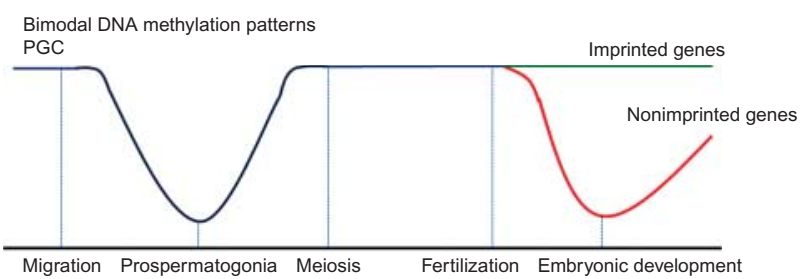

B

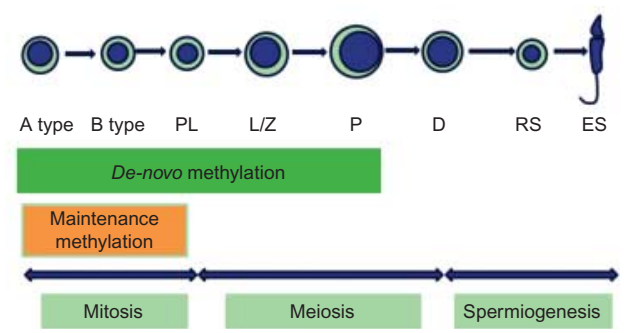

Figure 3 Schematic diagram reveals the expression of DNA methylation profiles in mammalian spermatogenesis. (A) Bimodal DNA methylation patterns in male germ cell development. PGCs are derived from epiblast at E6.5 and they migrate to the genital ridge. During the migration of PGCs, the epigenetic marks are widely erased. After the erasure of the DNA methylation markers, reestablishment of the male germ cell DNA patterns initiates from prospermatogonia to entering meiosis. After fertilization, DNA patterns are broadly erased by active demethylation activity, whereas the imprinted genes are maintained by DNMT1o activity. (B) The de-novo methylation and maintenance methylation activity initiates from type $A$ spermatogonia to meiosis. A type, type A spermatogonia; B type, type B spermatogonia; $\mathrm{PL}$, preleptotene spermatocytes; $\mathrm{P}$, pachytene spermatocytes; $\mathrm{D}$, diplotene spermatocytes; RS, round spermatids; ES, elongating spermatids.

the exception of the imprinted genes, which facilitate the expression of parent-specific genes in embryos. Finally, it has been demonstrated that the sperm and oocyte genomes have different demethylation modes during embryogenesis, and active DNA demethylation appears in parental genome compared to the replicationdependent demethylation in maternal genome (Santos et al. 2005).

\section{DNA methylation and SSC division and differentiation}

To maintain the stem cell pool, SSCs either remain in a quiescent state or undergo self-renewal. DNA methyltransferase 3-like (DNMT3L) is involved in SSC quiescence. DNMT3L positively regulates the stability of promyelocytic leukemia zinc finger (PLZF) in $\mathrm{THY} 1^{+}$ SSCs through down-regulating CDK2 expression, which may degrade PLZF through the ubiquitin pathway (Liao et al. 2014). The distal CpG island of Cdk2 promoter has been shown to be hypomethylated in Dnmt3I-knockout SSCs compared to the wide-type cells (Liao et al. 2014). On the other hand, it has been reported that the expression of DNMT3a2 and DNMT3b is undetectable in PLZF-positive and KIT-negative SSCs (Shirakawa et al. 2013). Thus, there are distinctive roles for different 
members of DNMT3 subfamily in regulating mitotic self-renewal of mouse SSCs.

Besides undergoing self-renewal, SSCs also give rise to differentiated spermatogonia. DNA methylation may be required to the transition from the Kit-negative SSCs to Kit-positive differentiated spermatogonia. It has been demonstrated that the transcripts of DNMT3a2 and DNMT3b remains at the highest level in type $A$ spermatogonia compared to other types of male germ cells. Shirakawa et al. (2013) have recently reported that DNMT3a2 and DNMT3b are not present in PLZFpositive and KIT-negative SSCs. Conversely, DNMT3a2 and DNMT3b expression is parallel with the Kit expression in spermatogonia. These observations suggest that DNA methylation is less prevalent in SSCs vs differentiated spermatogonia. Furthermore, overexpression of DNMT3b in the Kit-negative SSCs can induce Kit expression (Shirakawa et al. 2013). Altogether, the DNMT3a2 and DNMT3b may methylate genes essential for maintaining SSCs in an undifferentiated state and facilitating the transition from the undifferentiated spermatogonia to the differentiated state. It is of great interest to further explore the interaction between the DNA methylation dynamics and SSC differentiation in a global scale, which can shed light on the epigenetic causes of the non-obstructive azoospermia with spermatogenesis arrest in the SSC phase.

\section{The role of DNA methylation on meiosis and spermiogenesis}

The de novo DNA methylation and maintenance methylation are associated with meiosis in male germ cells, as illustrated by the observation that the expression of DNMT1, DNMT3a2 and DNMT3b is up-regulated in leptotene and zygotene spermatocytes (Oakes et al. 2007). The methylation of testis-specific genes PRM1 and PRM2 has been shown in meiotic prophase I (Trasler et al. 1990), and the imprinted genes and non-CpG island sequences are methylated in meiosis (Oakes et al. 2007). Demethylation of testis-specific genes may be required for meiotic initiation, since DNA methyltransferases are attenuated significantly in type B spermatogonia and preleptotene spermatocytes. Recently it has been reported that down-regulation of DNMT3L may increase the expression of the premeioticspecific gene-STRA8, leading to the onset of meiosis prematurely (Vanhoutteghem et al. 2014). In addition, it has been revealed that $5 \mathrm{mC}$-specific dioxygenase (TET1) can mediate the demethylation of the locus-specific genes, including the meiotic genes, and promotes the meiotic initiation through the activation of these genes in oocytes (Yamaguchi et al. 2012). It remains to be determined whether the function of TET1 in spermatogenesis resembles oocytes. Nevertheless, it is tempting to speculate that the meiotic initiator may be demethylated and activated in spermatogonia and preleptotene spermatocytes. Overexpression of DNMT3a2 and DNMT3b during this phase or knockout of TET1, Aid and Apobec 1 can be helpful to identify the essential factors in meiotic initiation.

In mice, the expression of DNMT1, DNMT3a2 and DNMT3b is enhanced in leptotene and zygotene spermatocytes and reduced in pachytene spermatocytes (La Salle \& Trasler 2006). In contrast to rodents, the expression of DNMT1, DNMT3a2 and DNMT3b peaks in pachytene spermatocytes in humans (Marques et al. 2011). These differences in the expression of DNMTs in rodent vs human male germ cells may result from the different isolation approaches used for separating these cells. The former utilized the STA-PUT method by cell gravity, while the latter employed the micromanipulation based upon the cellular diameters from testicular biopsy. However, the methylation activity in human pachytene spermatocytes is limited to the expression level and needs to be verified by functional assays. Additionally, the mechanisms that maintain epigenetic marks through meiosis especially in pachytene spermatocytes require additional studies and verification. Notably, during spermiogenesis in both mice and humans, DNMT1, DNMT3a2 and DNMT3b are highly expressed in round spermatids (La Salle \& Trasler 2006, Marques et al. 2011). Interestingly, DNMT1 is present in non-proliferative round spermatids whilst DNMT3a2 and DNMT3b are expressed after the establishment of paternal methylation pattern. Thus, DNMT3a2 and DNMT3b may function in maintaining the methylation patterns through the de novo methylation pathway, although the roles of DNMT1 in round spermatids remain to be elucidated in future studies.

\section{Summary}

In summary, we have discussed recent advances in the field regarding the roles of novel epigenetic regulators, including miRNAs and DNA methylation, in mitosis, meiosis and spermiogenesis. Although much progress has been made, there are many issues remaining to be investigated. First, more studies should be conducted in uncovering the spatiotemporal and sequential expression of these epigenetic regulators in male germ cells during spermatogenesis. Also, the roles of these epigenetic mediators in controlling spermatogenesis remain unknown. Secondly, it remains unclear which epigenetic regulators are the actual initiators for the onset of mitosis, meiosis and/or spermiogenesis. Thirdly, most of the information on the epigenetic regulators in spermatogenesis are derived from rodents, very little is known about epigenetic regulation on human spermatogenesis due to the difficulties in obtaining human testis tissues. Since cell types and stages of spermatogenesis are distinct between humans and rodents, it is essential to identify whether epigenetic regulators in rodent spermatogenesis are similar to humans. Recently it has 
been shown that human SSCs can be induced to differentiate into haploid spermatids which were used successfully for fertilization with full developmental capacity (Yang et al. 2014). It is interesting to explore what epigenetic regulators (miRNAs and DNA methylation) are involved in human SSC mitosis and differentiation. Finally, it is postulated that there are certain check points in the spermatogenesis, e.g. the progress from SSCs to differentiating spermatogonia, the entering of mitotic germ cells to meiotic process, and the transition from the canonical histone to the PRM in spermatids. During these critical processes, the interactions or crosstalk among epigenetic regulators remain unknown. Significantly, the stringent epigenetic regulation in spermatogenesis would yield novel insights into the etiology of sterility/infertility and offer new targets for gene therapy of male infertility. To uncover new epigenetic regulators on spermatogenesis will also facilitate the development of new approaches for male contraception since it is feasible to control the epigenetic regulation artificially without changing DNA sequences and the process is reversible and safe.

\section{Declaration of interest}

The authors declare that there is no conflict of interest that could be perceived as prejudicing the impartiality of the review reported.

\section{Funding}

This study was supported by key grants from the National Natural Science Foundation of China (31230048) and Chinese Ministry of Science and Technology (2014CB943101, 2013CB947901), grants from the National Natural Science Foundation of China (31171422, 31401250), the Program for Professor of Special Appointment (Eastern Scholar) at Shanghai Institutions of Higher Learning (2012.53), a key grant from the Science and Technology Commission of Shanghai Municipality (12JC1405900), Key Discipline and Specialty Foundation of Shanghai Municipal Commission of Health and Family Planning, and the Shanghai Pujiang Program (11PJ1406400).

\section{References}

Aravin AA \& Bourc'his D 2008 Small RNA guides for de novo DNA methylation in mammalian germ cells. Genes and Development 22 970-975. (doi:10.1101/gad.1669408)

Aravin AA, Hannon GJ \& Brennecke J 2007 The Piwi-piRNA pathway provides an adaptive defense in the transposon arms race. Science $\mathbf{3 1 8}$ 761-764. (doi:10.1126/science.1146484)

Bao J, Li D, Wang L, Wu J, Hu Y, Wang Z, Chen Y, Cao X, Jiang C, Yan W et al. 2012 MicroRNA-449 and microRNA-34b/c function redundantly in murine testes by targeting E2F transcription factor-retinoblastoma protein (E2F-pRb) pathway. Journal of Biological Chemistry 287 21686-21698. (doi:10.1074/jbc.M111.328054)

Bartel DP 2004 MicroRNAs: genomics, biogenesis, mechanism, and function. Cell 116 281-297. (doi:10.1016/S0092-8674(04)00045-5)
Bernstein BE, Mikkelsen TS, Xie X, Kamal M, Huebert DJ, Cuff J, Fry B, Meissner A, Wernig M, Plath K et al. 2006 A bivalent chromatin structure marks key developmental genes in embryonic stem cells. Cell $\mathbf{1 2 5}$ 315-326. (doi:10.1016/j.cell.2006.02.041)

Bjork JK, Sandqvist A, Elsing AN, Kotaja N \& Sistonen L 2010 miR-18, a member of Oncomir-1, targets heat shock transcription factor 2 in spermatogenesis. Development 137 3177-3184. (doi:10.1242/dev. 050955)

Bleckmann SC, Blendy JA, Rudolph D, Monaghan AP, Schmid W \& Schutz G 2002 Activating transcription factor 1 and CREB are important for cell survival during early mouse development. Molecular and Cellular Biology 22 1919-1925. (doi:10.1128/MCB.22.6.1919-1925. 2002)

Bostick M, Kim JK, Esteve PO, Clark A, Pradhan S \& Jacobsen SE 2007 UHRF1 plays a role in maintaining DNA methylation in mammalian cells. Science 317 1760-1764. (doi:10.1126/science.1147939)

Bouhallier F, Allioli N, Lavial F, Chalmel F, Perrard MH, Durand P, Samarut J, Pain B \& Rouault JP 2010 Role of miR-34c microRNA in the late steps of spermatogenesis. RNA 16 720-731. (doi:10.1261/rna. 1963810)

Boyer LA, Plath K, Zeitlinger J, Brambrink T, Medeiros LA, Lee TI, Levine SS, Wernig M, Tajonar A, Ray MK et al. 2006 Polycomb complexes repress developmental regulators in murine embryonic stem cells. Nature 441 349-353. (doi:10.1038/nature04733)

Brinster RL \& Avarbock MR 1994 Germline transmission of donor haplotype following spermatogonial transplantation. PNAS 91 11303-11307. (doi:10.1073/pnas.91.24.11303)

Buchold GM, Coarfa C, Kim J, Milosavljevic A, Gunaratne PH \& Matzuk MM 2010 Analysis of microRNA expression in the prepubertal testis. PLOS ONE 5 e15317. (doi:10.1371/journal.pone.0015317)

Cedar H \& Bergman Y 2009 Linking DNA methylation and histone modification: patterns and paradigms. Nature Reviews. Genetics 10 295-304. (doi:10.1038/nrg2540)

Cedar H \& Bergman Y 2012 Programming of DNA methylation patterns. Annual Review of Biochemistry 81 97-117. (doi:10.1146/annurevbiochem-052610-091920)

Chedin F, Lieber MR \& Hsieh CL 2002 The DNA methyltransferase-like protein DNMT3L stimulates de novo methylation by Dnmt3a. PNAS 99 16916-16921. (doi:10.1073/pnas.262443999)

Chen T \& Li E 2004 Structure and function of eukaryotic DNA methyltransferases. Current Topics in Developmental Biology 60 55-89. (doi:10.1016/S0070-2153(04)60003-2)

Chuang LS, lan HI, Koh TW, Ng HH, Xu G \& Li BF 1997 Human DNA(cytosine-5) methyltransferase-PCNA complex as a target for p21WAF1. Science 277 1996-2000. (doi:10.1126/science.277.5334.1996)

Cortellino S, Xu J, Sannai M, Moore R, Caretti E, Cigliano A, Le Coz M, Devarajan K, Wessels A, Soprano D et al. 2011 Thymine DNA glycosylase is essential for active DNA demethylation by linked deamination-base excision repair. Cell 146 67-79. (doi:10.1016/j.cell. 2011.06.020)

Dai L, Tsai-Morris CH, Sato H, Villar J, Kang JH, Zhang J \& Dufau ML 2011 Testis-specific miRNA-469 up-regulated in gonadotropin-regulated testicular RNA helicase (GRTH/DDX25)-null mice silences transition protein 2 and protamine 2 messages at sites within coding region: implications of its role in germ cell development. Journal of Biological Chemistry 286 44306-44318. (doi:10.1074/jbc.M111.282756)

Egger G, Liang G, Aparicio A \& Jones PA 2004 Epigenetics in human disease and prospects for epigenetic therapy. Nature 429 457-463. (doi:10.1038/nature02625)

Greenlee AR, Shiao MS, Snyder E, Buaas FW, Gu T, Stearns TM, Sharma M, Murchison EP, Puente GC \& Braun RE 2012 Deregulated sex chromosome gene expression with male germ cell-specific loss of Dicer1. PLOS ONE 7 e46359. (doi:10.1371/journal.pone.0046359)

Han J, Lee Y, Yeom KH, Kim YK, Jin H \& Kim VN 2004 The Drosha-DGCR8 complex in primary microRNA processing. Genes and Development 18 3016-3027. (doi:10.1101/gad.1262504)

Hashimoto H, Horton JR, Zhang X, Bostick M, Jacobsen SE \& Cheng X 2008 The SRA domain of UHRF1 flips 5-methylcytosine out of the DNA helix. Nature 455 826-829. (doi:10.1038/nature07280)

Hayashi K, Chuva de Sousa Lopes SM, Kaneda M, Tang F, Hajkova P, Lao K, O'Carroll D, Das PP, Tarakhovsky A, Miska EA et al. 2008 MicroRNA 
biogenesis is required for mouse primordial germ cell development and spermatogenesis. PLOS ONE 3 e1738. (doi:10.1371/journal.pone. 0001738)

He YF, Li BZ, Li Z, Liu P, Wang Y, Tang Q, Ding J, Jia Y, Chen Z, Li L et al. 2011 Tet-mediated formation of 5-carboxylcytosine and its excision by TDG in mammalian DNA. Science 333 1303-1307. (doi:10.1126/ science.1210944)

He Z, Jiang J, Kokkinaki M, Tang L, Zeng W, Gallicano I, Dobrinski I \& Dym M 2013 MiRNA-20 and mirna-106a regulate spermatogonial stem cell renewal at the post-transcriptional level via targeting STAT3 and Ccnd1. Stem Cells 31 2205-2217. (doi:10.1002/stem.1474)

Holliday R \& Pugh JE 1975 DNA modification mechanisms and gene activity during development. Science 187 226-232. (doi:10.1126/ science.1111098)

Huszar JM \& Payne CJ 2013 MicroRNA 146 (Mir146) modulates spermatogonial differentiation by retinoic acid in mice. Biology of Reproduction 88 15. (doi:10.1095/biolreprod.112.103747)

Hutvagner G, McLachlan J, Pasquinelli AE, Balint E, Tuschl T \& Zamore PD 2001 A cellular function for the RNA-interference enzyme Dicer in the maturation of the let-7 small temporal RNA. Science 293 834-838. (doi:10.1126/science.1062961)

Ito S, Shen L, Dai Q, Wu SC, Collins LB, Swenberg JA, He C \& Zhang Y 2011 Tet proteins can convert 5-methylcytosine to 5-formylcytosine and 5-carboxylcytosine. Science 333 1300-1303. (doi:10.1126/science. 1210597)

Jia D, Jurkowska RZ, Zhang X, Jeltsch A \& Cheng X 2007 Structure of Dnmt3a bound to Dnmt3L suggests a model for de novo DNA methylation. Nature 449 248-251. (doi:10.1038/nature06146)

Jones PA 1999 The DNA methylation paradox. Trends in Genetics 15 34-37. (doi:10.1016/S0168-9525(98)01636-9)

Jones PA 2012 Functions of DNA methylation: islands, start sites, gene bodies and beyond. Nature Reviews. Genetics 13 484-492. (doi:10.1038/nrg3230)

Jones PA \& Liang G 2009 Rethinking how DNA methylation patterns are maintained. Nature Reviews. Genetics 10 805-811. (doi:10.1038/ nrg2651)

Jung YH, Gupta MK, Shin JY, Uhm SJ \& Lee HT 2010 MicroRNA signature in testes-derived male germ-line stem cells. Molecular Human Reproduction 16 804-810. (doi:10.1093/molehr/gaq058)

Kagiwada S, Kurimoto K, Hirota T, Yamaji M \& Saitou M 2013 Replicationcoupled passive DNA demethylation for the erasure of genome imprints in mice. EMBO Journal 32 340-353. (doi:10.1038/emboj.2012.331)

Kanatsu-Shinohara M \& Shinohara T 2013 Spermatogonial stem cell selfrenewal and development. Annual Review of Cell and Developmental Biology 29 163-187. (doi:10.1146/annurev-cellbio-101512-122353)

Kelly TL, Li E \& Trasler JM 2003 5-aza-2'-deoxycytidine induces alterations in murine spermatogenesis and pregnancy outcome. Journal of Andrology 24 822-830.

Khaira H, McLean D, Ohl DA \& Smith GD 2005 Spermatogonial stem cell isolation, storage, and transplantation. Journal of Andrology 26 442-450. (doi:10.2164/jandrol.05062)

Korhonen HM, Meikar O, Yadav RP, Papaioannou MD, Romero Y, Da Ros M, Herrera PL, Toppari J, Nef S \& Kotaja N 2011 Dicer is required for haploid male germ cell differentiation in mice. PLOS ONE 6 e24821. (doi:10.1371/journal.pone.0024821)

Kuramochi-Miyagawa S, Watanabe T, Gotoh K, Totoki Y, Toyoda A, Ikawa M, Asada N, Kojima K, Yamaguchi Y, ljiri TW et al. 2008 DNA methylation of retrotransposon genes is regulated by Piwi family members MILI and MIWI2 in murine fetal testes. Genes and Development 22 908-917. (doi:10.1101/gad.1640708)

La Salle S \& Trasler JM 2006 Dynamic expression of DNMT3a and DNMT3b isoforms during male germ cell development in the mouse. Developmental Biology 296 71-82. (doi:10.1016/j.ydbio.2006.04.436)

Law JA \& Jacobsen SE 2010 Establishing, maintaining and modifying DNA methylation patterns in plants and animals. Nature Reviews. Genetics 11 204-220. (doi:10.1038/nrg2719)

Lee RC, Feinbaum RL \& Ambros V 1993 The C. elegans heterochronic gene lin-4 encodes small RNAs with antisense complementarity to lin-14. Cell 75 843-854. (doi:10.1016/0092-8674(93)90529-Y)

Lee Y, Ahn C, Han J, Choi H, Kim J, Yim J, Lee J, Provost P, Radmark O, Kim $S$ et al. 2003 The nuclear RNase III Drosha initiates microRNA processing. Nature 425 415-419. (doi:10.1038/nature01957)
Liang X, Zhou D, Wei C, Luo H, Liu J, Fu R \& Cui S 2012 MicroRNA-34c enhances murine male germ cell apoptosis through targeting ATF1. PLoS ONE 7 e33861. (doi:10.1371/journal.pone.0033861)

Liao HF, Chen WS, Chen YH, Kao TH, Tseng YT, Lee CY, Chiu YC, Lee PL, Lin QJ, Ching YH et al. 2014 DNMT3L promotes quiescence in postnatal spermatogonial progenitor cells. Development 141 2402-2413. (doi:10.1242/dev.105130)

Liu Y, Niu M, Yao C, Hai Y, Yuan Q, Liu Y, Guo Y, Li Z \& He Z 2015 Fractionation of human spermatogenic cells using STA-PUT gravity sedimentation and their miRNA profiling. Scientific Reports 58084. (doi:10.1038/srep08084)

Lund E, Guttinger S, Calado A, Dahlberg JE \& Kutay U 2004 Nuclear export of microRNA precursors. Science 303 95-98. (doi:10.1126/science. 1090599)

Maatouk DM, Loveland KL, McManus MT, Moore K \& Harfe BD 2008 Dicer1 is required for differentiation of the mouse male germline. Biology of Reproduction 79 696-703. (doi:10.1095/biolreprod.108. 067827)

Marques CJ, Joao Pinho M, Carvalho F, Bieche I, Barros A \& Sousa M 2011 DNA methylation imprinting marks and DNA methyltransferase expression in human spermatogenic cell stages. Epigenetics 6 1354-1361. (doi:10.4161/epi.6.11.17993)

Mayer W, Niveleau A, Walter J, Fundele R \& Haaf T 2000 Demethylation of the zygotic paternal genome. Nature 403 501-502. (doi:10.1038/ 35000656)

Mertineit C, Yoder JA, Taketo T, Laird DW, Trasler JM \& Bestor TH 1998 Sex-specific exons control DNA methyltransferase in mammalian germ cells. Development 125 889-897.

Messerschmidt DM, Knowles BB \& Solter D 2014 DNA methylation dynamics during epigenetic reprogramming in the germline and preimplantation embryos. Genes and Development 28 812-828. (doi:10.1101/gad.234294.113)

Moritoki Y, Hayashi Y, Mizuno K, Kamisawa H, Nishio H, Kurokawa S, Ugawa S, Kojima Y \& Kohri K 2014 Expression profiling of microRNA in cryptorchid testes: miR-135a contributes to the maintenance of spermatogonial stem cells by regulating FoxO1. Journal of Urology 191 1174-1180. (doi:10.1016/j.juro.2013.10.137)

Nguyen CT, Gonzales FA \& Jones PA 2001 Altered chromatin structure associated with methylation-induced gene silencing in cancer cells: correlation of accessibility, methylation, MeCP2 binding and acetylation. Nucleic Acids Research 29 4598-4606. (doi:10.1093/nar/29.22.4598)

Niu Z, Goodyear SM, Rao S, Wu X, Tobias JW, Avarbock MR \& Brinster RL 2011 MicroRNA-21 regulates the self-renewal of mouse spermatogonial stem cells. PNAS 108 12740-12745. (doi:10.1073/pnas.1109987108)

Novotny GW, Sonne SB, Nielsen JE, Jonstrup SP, Hansen MA, Skakkebaek NE, Rajpert-De Meyts E, Kjems J \& Leffers H 2007 Translational repression of E2F1 mRNA in carcinoma in situ and normal testis correlates with expression of the miR-17-92 cluster. Cell Death and Differentiation 14 879-882. (doi:10.1038/sj.cdd.4402090)

Okano M, Xie S \& Li E 1998 Cloning and characterization of a family of novel mammalian DNA (cytosine-5) methyltransferases. Nature Genetics 19 219-220. (doi:10.1038/890)

Oakes CC, La Salle S, Smiraglia DJ, Robaire B \& Trasler JM 2007 Developmental acquisition of genome-wide DNA methylation occurs prior to meiosis in male germ cells. Developmental Biology 307 368-379. (doi:10.1016/j.ydbio.2007.05.002)

Ooi SK, Qiu C, Bernstein E, Li K, Jia D, Yang Z, Erdjument-Bromage H, Tempst P, Lin SP, Allis CD et al. 2007 DNMT3L connects unmethylated lysine 4 of histone $\mathrm{H} 3$ to de novo methylation of DNA. Nature $\mathbf{4 4 8}$ 714-717. (doi:10.1038/nature05987)

Oswald J, Engemann S, Lane N, Mayer W, Olek A, Fundele R, Dean W, Reik W \& Walter J 2000 Active demethylation of the paternal genome in the mouse zygote. Current Biology 10 475-478. (doi:10.1016/S09609822(00)00448-6)

Pan G, Tian S, Nie J, Yang C, Ruotti V, Wei H, Jonsdottir GA, Stewart R \& Thomson JA 2007 Whole-genome analysis of histone $\mathrm{H} 3$ lysine 4 and lysine 27 methylation in human embryonic stem cells. Cell Stem Cell $\mathbf{1}$ 299-312. (doi:10.1016/j.stem.2007.08.003)

Papaioannou MD, Pitetti JL, Ro S, Park C, Aubry F, Schaad O, Vejnar CE, Kuhne F, Descombes P, Zdobnov EM et al. 2009 Sertoli cell Dicer is essential for spermatogenesis in mice. Developmental Biology 326 250-259. (doi:10.1016/j.ydbio.2008.11.011) 
Persengiev SP \& Green MR 2003 The role of ATF/CREB family members in cell growth, survival and apoptosis. Apoptosis 8 225-228. (doi:10.1023/ A:1023633704132)

Popp C, Dean W, Feng S, Cokus SJ, Andrews S, Pellegrini M, Jacobsen SE \& Reik W 2010 Genome-wide erasure of DNA methylation in mouse primordial germ cells is affected by AID deficiency. Nature 463 1101-1105. (doi:10.1038/nature08829)

Portela A \& Esteller M 2010 Epigenetic modifications and human disease. Nature Biotechnology 28 1057-1068. (doi:10.1038/nbt.1685)

Pradhan S, Bacolla A, Wells RD \& Roberts RJ 1999 Recombinant human DNA (cytosine-5) methyltransferase. I. Expression, purification, and comparison of de novo and maintenance methylation. Journal of Biological Chemistry 274 33002-33010. (doi:10.1074/jbc.274.46. 33002)

Rai K, Huggins IJ, James SR, Karpf AR, Jones DA \& Cairns BR 2008 DNA demethylation in zebrafish involves the coupling of a deaminase, a glycosylase, and gadd45. Cell 135 1201-1212. (doi:10.1016/j.cell.2008. 11.042)

Ro S, Park C, Sanders KM, McCarrey JR \& Yan W 2007 Cloning and expression profiling of testis-expressed microRNAs. Developmental Biology 311 592-602. (doi:10.1016/j.ydbio.2007.09.009)

Romero Y, Meikar O, Papaioannou MD, Conne B, Grey C, Weier M, Pralong F, De Massy B, Kaessmann H, Vassalli JD et al. 2011 Dicer1 depletion in male germ cells leads to infertility due to cumulative meiotic and spermiogenic defects. PLOS ONE 6 e25241. (doi:10.1371/journal. pone.0025241)

Sadri-Ardekani H, Mizrak SC, van Daalen SK, Korver CM, RoepersGajadien HL, Koruji M, Hovingh S, de Reijke TM, de la Rosette JJ, van der Veen F et al. 2009 Propagation of human spermatogonial stem cells in vitro. Journal of American Medical Association 302 2127-2134. (doi:10.1001/jama.2009.1689)

Saitou M \& Yamaji M 2012 Primordial germ cells in mice. Cold Spring Harbor Perspectives in Biology 4 pii: a008375. (doi:10.1101/cshperspect.a008375)

Santos F, Peters AH, Otte AP, Reik W \& Dean W 2005 Dynamic chromatin modifications characterise the first cell cycle in mouse embryos. Developmental Biology 280 225-236. (doi:10.1016/j.ydbio. 2005.01.025)

Seisenberger S, Andrews S, Krueger F, Arand J, Walter J, Santos F, Popp C, Thienpont B, Dean W \& Reik W 2012 The dynamics of genome-wide DNA methylation reprogramming in mouse primordial germ cells. Molecular Cell 48 849-862. (doi:10.1016/j.molcel.2012.11.001)

Shirakawa T, Yaman-Deveci R, Tomizawa S, Kamizato Y, Nakajima K, Sone H, Sato Y, Sharif J, Yamashita A, Takada-Horisawa Y et al. 2013 An epigenetic switch is crucial for spermatogonia to exit the undifferentiated state toward a Kit-positive identity. Development 140 3565-3576. (doi:10.1242/dev.094045)

Song J, Teplova M, Ishibe-Murakami S \& Patel DJ 2012 Structure-based mechanistic insights into DNMT1-mediated maintenance DNA methylation. Science 335 709-712. (doi:10.1126/science.1214453)

Takai D \& Jones PA 2002 Comprehensive analysis of CpG islands in human chromosomes 21 and 22. PNAS 99 3740-3745. (doi:10.1073/pnas. 052410099)

Tang F, Kaneda M, O'Carroll D, Hajkova P, Barton SC, Sun YA, Lee C, Tarakhovsky A, Lao K \& Surani MA 2007 Maternal microRNAs are essential for mouse zygotic development. Genes and Development 21 644-648. (doi:10.1101/gad.418707)

Thomson JP, Skene PJ, Selfridge J, Clouaire T, Guy J, Webb S, Kerr AR, Deaton A, Andrews R, James KD et al. 2010 CpG islands influence chromatin structure via the CpG-binding protein Cfp1. Nature $\mathbf{4 6 4}$ 1082-1086. (doi:10.1038/nature08924)

Tong MH, Mitchell D, Evanoff R \& Griswold MD 2011 Expression of Mirlet7 family microRNAs in response to retinoic acid-induced spermatogonial differentiation in mice. Biology of Reproduction 85 189-197. (doi:10. 1095/biolreprod.110.089458)
Tong MH, Mitchell DA, McGowan SD, Evanoff R \& Griswold MD 2012 Two miRNA clusters. Mir-17-92 (Mirc1) and Mir-106b-25 (Mirc3), are involved in the regulation of spermatogonial differentiation in mice. Biology of Reproduction 86 72. (doi:10.1095/biolreprod.111.096313)

Trasler JM, Hake LE, Johnson PA, Alcivar AA, Millette CF \& Hecht NB 1990 DNA methylation and demethylation events during meiotic prophase in the mouse testis. Molecular and Cellular Biology 10 1828-1834.

Tscherner A, Gilchrist G, Smith N, Blondin P, Gillis D \& LaMarre J 2014 MicroRNA-34 family expression in bovine gametes and preimplantation embryos. Reproductive Biology and Endocrinology 12 85. (doi:10.1186/ 1477-7827-12-85)

Vanhoutteghem A, Messiaen S, Herve F, Delhomme B, Moison D, Petit JM, Rouiller-Fabre V, Livera G \& Djian P 2014 The zinc-finger protein basonuclin 2 is required for proper mitotic arrest, prevention of premature meiotic initiation and meiotic progression in mouse male germ cells. Development 141 4298-4310. (doi:10.1242/dev. 112888)

Wightman B, Ha I \& Ruvkun G 1993 Posttranscriptional regulation of the heterochronic gene lin-14 by lin-4 mediates temporal pattern formation in C. elegans. Cell 75 855-862. (doi:10.1016/0092-8674 (93)90530-4)

Wu Q, Song R, Ortogero N, Zheng H, Evanoff R, Small CL, Griswold MD, Namekawa SH, Royo H, Turner JM et al. 2012 The RNase III enzyme DROSHA is essential for microRNA production and spermatogenesis. Journal of Biological Chemistry 287 25173-25190. (doi:10.1074/jbc. M112.362053)

Wu J, Bao J, Kim M, Yuan S, Tang C, Zheng H, Mastick GS, Xu C \& Yan W 2014 Two miRNA clusters, miR-34b/c and miR-449, are essential for normal brain development, motile ciliogenesis, and spermatogenesis. PNAS 111 E2851-E2857. (doi:10.1073/pnas.1407777111)

Yamaguchi S, Hong K, Liu R, Shen L, Inoue A, Diep D, Zhang K \& Zhang Y 2012 Tet1 controls meiosis by regulating meiotic gene expression. Nature 492 443-447. (doi:10.1038/nature11709)

Yang QE, Racicot KE, Kaucher AV, Oatley MJ \& Oatley JM 2013 MicroRNAs 221 and 222 regulate the undifferentiated state in mammalian male germ cells. Development 140 280-290. (doi:10. 1242/dev.087403)

Yang S, Ping P, Ma M, Li P, Tian R, Yang H, Liu Y, Gong Y, Zhang Z, Li Z et al. 2014 Generation of haploid spermatids with fertilization and development capacity from human spermatogonial stem cells of cryptorchid patients. Stem Cell Reports 3 663-675. (doi:10.1016/j.stemcr.2014. 08.004)

Yu Z, Raabe NB \& Hecht NB 2005 Mirn122a reduces expression of the posttranscriptionally regulated germ cell transition protein 2 (Tnp2) messenger RNA (mRNA) by mRNA cleavage. Biology of Reproduction 73 427-433. (doi:10.1095/biolreprod.105.040998)

Yu M, Mu H, Niu Z, Chu Z, Zhu H \& Hua J 2014 miR-34c enhances mouse spermatogonial stem cells differentiation by targeting Nanos2. Journal of Cellular Biochemistry 115 232-242. (doi:10.1002/jcb.24655)

Zimmermann C, Romero Y, Warnefors M, Bilican A, Borel C, Smith LB, Kotaja N, Kaessmann H \& Nef S 2014 Germ cell-specific targeting of DICER or DGCR8 reveals a novel role for endo-siRNAs in the progression of mammalian spermatogenesis and male fertility. PLOS ONE 9 e107023. (doi:10.1371/journal.pone.0107023)

Received 9 December 2014

First decision 30 January 2015

Revised manuscript received 26 March 2015

Accepted 7 April 2015 